\title{
Ariane et Barbe-Bleue de Maeterlinck et l'apport symboliste
}

Zöllner, R

\begin{abstract}
Maurice Maeterlinck oriente la tradition de Barbe-Bleue dans plusieurs directions inédites : il la rattache à d'autres mythèmes, surtout en la combinant avec la figure d'Ariane ; et il adapte des éléments traditionnels (les premières femmes ne sont pas mortes mais prisonnières) ou en développe d'autres à la lueur de l'esthétique symboliste (couleurs, portes et pierreries). La curiosité féminine que réprouve Perrault avec un clin d'oeil, devient une véritable quête du savoir. Ariane et Barbe-Bleue est régi par une structure binaire : une double délivrance est précédée de deux transgressions. Comme le symbolisent les deux clés, la pièce montre le caractère multifacétique du masculin et du féminin : une Ariane femme forte, progressiste, contraste avec les autres femmes anxieuses et faibles. Barbe-Bleue, sans barbe et captif à la fin, l'emporte dans la mesure où il arrive à retenir les premières femmes. La pièce de Maeterlinck peut être considérée comme une oeuvre clé qui ouvre la voie aux adaptations modernes du XXe siècle.
\end{abstract}

DOI: https://doi.org/10.1515/fabula-2013-0005

Posted at the Zurich Open Repository and Archive, University of Zurich

ZORA URL: https://doi.org/10.5167/uzh-86746

Journal Article

Published Version

Originally published at:

Zöllner, R (2013). Ariane et Barbe-Bleue de Maeterlinck et l'apport symboliste. Fabula, 54(1-2):46-60. DOI: https://doi.org/10.1515/fabula-2013-0005 
Reto Zö1lner, Zürich

\title{
Ariane et Barbe-Bleue de Maeterlinck et l'apport symboliste
}

\author{
Au carrefour des mythes et des femmes
}

\begin{abstract}
Assurément, Ariane et Barbe-Bleue (1901) ${ }^{1}$ est plus que ce petit jeu de scène sans grande arrière-pensée morale ou philosophique dont Maeterlinck - obéissant à un mouvement de captatio benevolentiae ? - parle dans la préface ${ }^{2}$. Qu'il s'intéresse au mythe de la Barbe-Bleue ne peut, vu l'orientation que prend son auvre, nullement étonner. Depuis toujours séduit par tout ce qui émane de la culture populaire (ses Chansons le disent déjà assez) et du Märchen (telle que La Princesse Maleine, qui s'inspire entre autre du conte Prinzessin Maleen des frères $\mathrm{Grimm}^{3}$ ), il découvre dans Barbe-Bleue des composants-clé de son esthétique théâtrale, une esthétique solidement affirmée depuis le succès retentissant de La Princesse Maleine ${ }^{4}$. Songeons, en effet, au mystère psychologique du héros éponyme, personnage délibérément opaque que Maeterlinck transpose, avec le caractère spectral des premières femmes de Barbe-Bleue, sur le plan des victimes ; en général, la « Sinnverdunkelung > (l’obscurcissement du sens) que postule W infried Menninghaus ${ }^{5}$ comme trait majeur de la tradition, avait tout pour fasciner Maeterlinck, imprégné fortement de la métaphysique d'un Ruysbroeck ou d'un
\end{abstract}

1 L'opéra éponyme de Paul Dukas date de 1907. Une version allemande (Blaubart und Ariane, oder die vergebliche Befreiung) paraît déjà en 1899 dans la "Wiener Rundschau » (traduction de F. von Oppeln-Bronikowski).

2 Maeterlinck, Maurice : Préface. Dans : Théâtre 1-3. Genève 1979, I-XVIII, ici XVIII.

3 cf. van de Kerckhove, Fabrice : Lecture. Dans : Maeterlinck, Maurice : La Princesse Maleine. éd. id. Bruxelles 1998, 249-287, ici 257-259. Déjà les premiers écrits de Maeterlinck sont placés sous le signe du conte : «Le Massacre des Innocents » (1886, qui s'inspire d'un tableau de Breughel), « Onirologie » (1889) et un peu plus tard «L'Anneau de Polycrate » (1893).

4 La parution de «La Princesse Maleine » (1890), signalée par Octave Mirbeau comme l'œuvre la plus géniale de son temps, comparable à Shakespeare (!), deviendra un événement majeur. Le monde littéraire tourne brusquement ses yeux sur Maeterlinck, confronté à une subite et inespérée publicité. Suit - mis à part les essais - une phase imposante de production théâtrale : sortiront en deux ans «L'Intruse », «Les Aveugles », «Les sept Princesses » et «Pelléas et Mélisande ».

5 Menninghaus, Winfried : Lob des Unsinns : über Kant, Tieck und Blaubart. Frankfurt am Main 1995, 234. 
Novalis ${ }^{6}$. Si Paul Gorceix, en dehors de la poésie du mystère ${ }^{7}$, voit l'univers dramatique maeterlinckien dominé par la triade de la mort ${ }^{8}$, de 1 'amour et de la peur', il n'y a qu'un petit pas à franchir pour plonger dans le monde 〈 bleu > de la mort mystérieuse des femmes, de l'amour atroce de Barbe-Bleue et des menaces angoissantes au sujet de la porte défendue. La porte, au demeurant, joue un rôle capital dans le symbolisme maeterlinckien. Le mystère du secret et des portes fermées dans Ariane et Barbe-Bleue répond par exemple, dans Pelléas et Mélisande, au souci de clarté et au désir exprimé par les protagonistes que les portes doivent rester toujours ouvertes ${ }^{10}$.

Qu'en est-il, cependant, de la tradition de Barbe-Bleue ? Les changements principaux par rapport à Perrault - croiser Barbe-Bleue avec le mythe d'Ariane en opposant une femme forte tant à la force occulte du mari qu'à la faiblesse des premières épouses, laisser en vie celles-ci, chez Maeterlinck simplement captives, et surtout créer une fin tragique - soulignent l'originalité d'Ariane et Barbe-Bleue. La tentative de cette contribution est alors d'étudier quelques aspects de la vision maeterlinckienne de Barbe-Bleue et de cerner en quoi consiste sa propre version, résolument moderne ${ }^{11}$.

\section{Le personnage d'Ariane ou le croisement des mythes}

La nouveauté la plus saillante de la version maeterlinckienne est sans aucun doute l'intrusion (dans le titre !) de la figure d'Ariane, dont la valeur s'avère multiple et non uniquement liée à la teneur progressiste, voire féministe du texte, comme l'on a tendance à le résumer trop sommairement ${ }^{12}$.

6 Renvoyons à Maurice Maeterlinck, « Ruysbroeck l'Admirable » (1889), sa traduction (1891) de "L'Ornement des noces spirituelles » par Ruysbroeck et au chapitre sur Novalis dans « Le Trésor des humbles » (1896).

7 Gorceix, Paul : Maeterlinck ou la poésie du mystère. Dans : Modernités 14 (2001) 221-244.

8 Maeterlinck parle lui-même d'une « petite trilogie de la mort » que forment les pièces «L'Intruse », « Les Aveugles » et « Les sept Princesses ».

9 Gorceix, Paul : Les Affinités allemandes dans l'œuvre de Maurice Maeterlinck. Poitiers $1975,210$.

${ }^{10}$ Voir à ce sujet Postic, Marcel : Maeterlinck et le Symbolisme. Paris 1970, 122. Maeterlinck est donc véritablement hanté par le symbole de la porte, ce dont témoignent aussi d'autres pièces telles que « La Mort de Tintagiles », «L'Intruse » ou « La Princesse Maleine ".

${ }^{11}$ Étudier les apports et changements de Maeterlinck par rapport à la tradition de BarbeBleue revient toujours à une comparaison plus ou moins directe avec le conte de Perrault, laquelle, non seulement pour les versions françaises, mais aussi pour la racine germanique des frères Grimm, a joué le rôle de modèle (cf. Szczepaniak, Monika : Männer in Blau. Blaubart-Bilder in der deutschsprachigen Literatur. Köln 2005, 95). Très généralement, l'énorme distance de Maeterlinck vis-à-vis de Perrault se mesure dans le cadre onirique, à l'antipode du réalisme perraultien.

${ }^{12}$ Voir, par exemple, Knapp, Bettina : Maurice Maeterlinck. Washington 1975, 100; Caswell, Austin B. : Maeterlinck's et Dukas's « Ariane et Barbe-Bleue » : A Feminist Opera ? Dans : Studies in Romanticism 27,2 (1988) 203-221. 


\subsection{Musicalité et onomastique}

Partant d'une perspective génétique, Ariane fait penser, quant à l'onomastique, à Anne, la sœur de la dernière épouse chez Perrault et à un des noms propres plutôt rares du conte. L'insistance sur le nom d'Anne lui confère quasiment le statut d'un appel à l'aide («Anne, ma sœur Anne, ne vois-tu rien venir ? )). Elle crée une de ces répétitions typiques du conte dont s'inspire également l'écriture poéticodramatique de Maurice Maeterlinck, favorisée encore par sa forte tendance à la stichomythie. Si, chez Perrault, Anne annonce le secours prochain que promettent les deux cavaliers-frères, Ariane n'est ni messagère ni dépendante d'adjuvants masculins. Elle entreprend au contraire elle-même l'acte de la délivrance non seulement des femmes captives, mais aussi, à la fin de l'acte III, de Barbe-Bleue in persona.

On pourrait relever de plus une autre dimension des noms, car < Ariane > (/ a R a /) s'accorde de façon remarquable à < Barbe-Bleue > (/ b a R b /), un effet phonique qui n'a certainement pas échappé à Maeterlinck, toujours très sensible à de tels jeux de rappels. Après le célèbre dicton de Verlaine qui fait date (« de la musique avant toute chose ») et les réflexions mallarméennes, la musicalité soit des vers, soit de la prose poétique, est une pierre de touche de l'esthétique symboliste en général, du symbolisme belge ${ }^{13}$ et de Maeterlinck en particulier. Un des exemples les plus illustratifs de ce qu'on pourrait appeler avec Gérard Dessons la logique des séries prosodiques ${ }^{14}$, est peut-être celui de La Princesse Maleine, où l'onomastique et la répétition phonique en assonances et allitérations réfèrent directement au destin de la dramatis personae : «Maleine m'a l'air un peu malade $»^{15}$. Voilà, pour revenir à Ariane et Barbe-Bleue, un heurt quasiment mythique entre les deux lettres initiales de l'alphabet, mimant la lutte des deux forces masculine et féminine. Le lien fatidique entre Barbe-Bleue et Ariane et, à première vue, leur relation hiérarchique, se liraient alors au niveau des noms - nomina sunt consequentia rerum : Ariane, victorieuse, est placée avant et devant Barbe-Bleue.

\subsection{Au carrefour des mythes}

A la dimension musicale et onomastique s'ajoute, bien sûr, celle de la mytho$\operatorname{logi\mathrm {e}^{16}}$. Maeterlinck exploite surtout deux thèmes associés à la figure d'Ariane, à savoir la délivrance guidée et l'abandon.

Comme la dernière femme de Barbe-Bleue, son pendant mythologique, dénouant le fil du peloton de laine, ouvre une porte dangereuse, celle précisément qui mène

${ }^{13}$ Voir par exemple l'essai intitulé « Traité du Verbe » (1886) de René Ghil.

${ }^{14}$ Dessons, Gérard : Maeterlinck. Le théâtre du poème. Paris 2005, 93.

${ }^{15} \mathrm{cf}$. Maeterlinck, «La Princesse Maleine », acte III, scène 3 (voir note 3).

${ }^{16}$ Pour les détails sur la figure mythologique d'Ariane cf. Ranke-Graves, Robert : Griechische Mythologie. Quellen und Deutung. Hamburg 2003. 
à la chambre du Minotaure. Les clefs données aux femmes de Barbe-Bleue, qui les jettent dans le malheur, font alors écho au fil qui sauve Thésée. Tandis qu'Ariane guide le héros grec à travers le labyrinthe du Minotaure, l'Ariane maeterlinckienne ramène les femmes à travers la grotte souterraine vers la liberté, ce qui fait, par ailleurs, de Barbe-Bleue un autre Minotaure insatiable, dévorant comme victimes ses propres femmes. L'aspect de l'abandon, en revanche, est radicalement inversé par Maeterlinck. Si Thésée abandonne Ariane sur l'île de Naxos, c'est bien Ariane qui, voyant échouer sa mission (séparer les autres femmes de Barbe-Bleue), parvient, chez le poète gantois, à se libérer de l'emprise de l'époux. D'une Ariane passive, figure du délaissement, renaît une Ariane femme forte et dominant les relations intersexuelles, mais condamnée toutefois à rester un être d'exception apparemment incapable de servir de modèle pour les premières femmes.

Par surcroît, il existe, ce qui est rappelé par un emprunt direct dans le texte maeterlinckien, un lien entre Ariane et le mythe de Phèdre. Phèdre et Ariane sont les deux filles de Pasiphaé, dont le lignage remontant à Hélios-Sol est hanté par Vénus. Au même degré que sa soeur Phèdre, Ariane est donc placée sous l'enseigne de l'amour malheureux. Qu'elle tombe entre les mains de Barbe-BleueMinautore ne fait que sceller le fatal héritage familial. L'hémistiche maeterlinckien « n'allez pas plus avant» (M 131 $\left.{ }^{17}\right)$ joue en effet sur une citation de Phèdre : «N'allons point plus avant. Demeurons, chère Enone »(v. 153). Il marque, comme chez Racine, un seuil qui ne devrait pas être franchi et éclaire in nuce le sort des deux filles de Pasiphaé ${ }^{18}$. Placé tout au début, cet hémistiche fait comme si la pièce s'ouvrait avec un destin inévitable, un drame d'amour funeste autour d'un homme incapable d'aimer autre chose qu'un modèle de fidélité et d'obéissance (par rapport auquel, nécessairement, toute femme est forcée d'échouer) et autour de l'impossibilité des épouses de sortir de l'emprise de Barbe-Bleue. Celui-ci ne tue pas ce qu'il aime, pour reprendre l'expression de Helmut Barz ${ }^{19}$. Comparable à Don Juan, il est incapable d'amour et ne semble considérer les femmes que comme une sorte d'échelle d'expérience morale ou comme moyen de satisfaire ses fantaisies cruelles ${ }^{20}$.

Le pessimisme maeterlinckien épouse le fatum intériorisé tout en quittant le tragique cosmique et prédéterminé de Phèdre. On pourrait avancer avec María Jesús Cabral que «Maeterlinck a donc su intervertir (terme fondamental de sa doctrine théâtrale) des notions dramaturgiques issues de la tragédie classique. Les

${ }^{17}$ Les indications de pages se réfèrent à Maeterlinck (voir note 2) t. 3.

${ }^{18}$ Le vers de « Phèdre » se situe à l'acte I, scène 3. Dans la tragédie racinienne, le seuil à franchir est, comme on le sait, l'aveu de l'amour coupable.

${ }^{19}$ Barz, Helmut : Blaubart : wenn einer vernichtet, was er liebt. Zürich 1987.

${ }^{20} \mathrm{La}$ recherche de l'absolu et d'un idéal (bleu) rapproche évidemment Barbe-Bleue de Don Juan. Voir à cet égard le livre de Wertheimer, Jürgen : Don Juan und Blaubart. Erotische Serientäter in der Literatur. München 1999. L'échec de l'amour se lit, tant pour Don Juan que pour Barbe-Bleue, dans la série des victimes qui ne peut être interrompue que par la mort du protagoniste. 
conflits sont intérieurs, la catastrophe est intime [...] $»^{21}$. L'allusion à Phèdre et à la tragédie classique ne trompe pas non plus au niveau formel. «N'allez pas plus avant » est exactement un hémistiche (d'un alexandrin), et l'on peut observer, à plusieurs reprises dans Ariane et Barbe-Bleue, une sorte de tentation de l'alexandrin pour la prose poétique de Maeterlinck, comme par exemple dans ce dialogue entre Ariane et Barbe-Bleue devant la porte fatidique : "C'était bien peu de chos(e) ce que je demandais / Vous leur demandiez plus que vous n'aviez donné / Vous perdez le bonheur que je voulais pour vous » (M 142). De manière analogue, il est aisé d'y relever des structures de rimes (touche - bouche; belle - rebelle, M 147).

Les renvois mythiques, il importe de le dire, ne s'arrêtent pas là. Ariane et BarbeBleue inclut et retravaille, outre Ariane et Phèdre, d'autres grands mythes et récits, dont par exemple :

\section{Le meurtre de César}

Ce grand épisode de l'histoire romaine est repris dans un pastiche du « tu quoque >, qui devient le «Vous aussi ... » (M 141) que Barbe-Bleue prononce sur le point d'attraper sa femme en flagrant délit devant la porte interdite. Ainsi pourrait-on comprendre les coups que la dernière femme porte à la volonté de Barbe-Bleue comme ceux portés à César. Reprenant la formule sous forme d'écho - si cher à Maeterlinck -, Ariane ne tarde pas à justifier ce ‘ meurtre > du mari-père à la manière de Brutus et réplique : 〈Moi surtout 〉. Culminant dans la transgression de l'ordre, le meurtre symbolique du mari côtoie celui, évidemment freudien, du père.

\section{La descente aux enfers}

Quelques critiques tels que Mererid Puw Davies associent Barbe-Bleue à Hadès ${ }^{22}$. Ce rapprochement se concrétise chez Maeterlinck avec la descente qu'entreprend Ariane dans la grotte souterraine afin de délivrer les autres femmes prisonnières de Hadès-Barbe-Bleue. Dans cette perspective, l'héroüne se mettrait dans le sillage d'Orphée ou, pour renouer avec Phèdre, du héros Thésée. De plus, le décor rappelant le locus amoenus qui attend les femmes au sortir de la prison, fait penser au retour saisonnier de Proserpine quittant le monde souterrain pour la terre.

${ }^{21}$ Cabral, María Jesús : Mallarmé hors frontières. Des défis de l'œuvre au filon symbolique du premier théâtre maeterlinckien. Amsterdam/New York 2007, 322.

${ }^{22}$ Davies, Mererid Puw : The Tale of Bluebeard in German Literature : from the Eighteenth Century to the Present. Oxford 2001, 76. 


\section{Jésus-Christ délivrant le peuple}

Ariane encourage les autres femmes à rejeter leur vie de terreur et à l'accompagner vers la liberté en utilisant, une fois de plus dans la forme majestueuse de l'alexandrin, l'expression de la < bonne nouvelle > dont témoignent les Évangiles : «Vous ne voulez pas croire à la bonne nouvelle » (M 151). Le désir d'Ariane de prendre toute seule ce dernier chemin de délivrance, après avoir renvoyé la nourrice, provoque les mêmes associations : «Je veux y aller seule » (M 139).

Force est de constater la même concentration, le même mouvement centrifuge, dans le théâtre maeterlinckien lui-même. Tous les noms des personnages féminins d'Ariane et Barbe-Bleue, c'est-à-dire ceux des cinq premières femmes, sont en effet empruntés à des pièces précédentes : Mélisande, bien évidemment, mais aussi Alladine (Alladine et Palomides), Sélysette (Aglavaine et Sélysette) ainsi que Ygraine et Bellangère (La Mort de Tintagiles). Dans cette "pièce allégorique ${ }^{23}$, seule Ariane porte un nom «non-maeterlinckien > et devient une vraie intruse qui se distingue - nomen est omen - foncièrement des autres épouses. Ariane et BarbeBleue se trouve alors à la fois au carrefour de certains grands mythes occidentaux et au carrefour de l'œuvre dramatique de Maurice Maeterlinck.

\section{La symbolique de la couleur: aller au-delà de l'azur}

\subsection{Le bleu et la chromatique symbolique}

Le personnage d'Ariane et l'opposition qu'il forme avec les cinq autres épouses captives étant sans doute un élément pilier chez Maeterlinck, sa réécriture de Barbe-Bleue peut comprendre de même le développement d'un élément déjà présent dans la tradition perraultienne. À titre d'exemple, ce chapitre s'intéressera au thème de la couleur. Si le bleu est généralement isolé, Maeterlinck crée autour de cette couleur < phare > tout un réseau de couleurs symboliques.

La barbe bleue est, chez Perrault, un détail «réaliste `. L'auteur des Contes lui attribue les caractéristiques d'une terrible laideur et de la peur qu'elle inspire, de sorte que tout mariage tiendrait de la gageure, n'était la richesse du futur mari. Dans Ariane et Barbe-Bleue, en revanche, la couleur bleue s'inscrit dans un jeu symbolique des couleurs. Pour la première fois à l'acte I, pendant lequel Ariane et la nourrice ouvrent successivement toutes les portes et découvrent à chaque fois une mer de pierres précieuses : améthystes, saphirs, perles, émeraudes, rubis et, pour finir, des diamants. Les couleurs diaprées des ces pierres précieuses renvoient de façon explicite aux éléments de la nature au moment où le cortège des femmes de Barbe-Bleue, guidé par Ariane, quitte la prison souterraine (acte II). D'une manière systématique, on peut donc mettre en parallèle la couleur d'une pierre

${ }^{23}$ Pourtois, Anne : Maeterlinck et la réalité des songes. Dans : Europe 40 (1962) 59-76, ici 73. 
précieuse à l'acte I, par exemple bleu ou vert, avec un élément de la nature à l'acte II, en l'ocurrence la mer, le ciel ou la campagne fleurie. Au niveau métaphorique, ce rapprochement est déjà annoncé à l'acte I où les saphirs sont comparés au ciel bleu, les émeraudes au printemps et au village natal (M 137), lesquels préfigurent en effet l'évocation de la campagne à l'acte II ; les améthystes comparées aux «violettes de miracles » (M 135) font penser de même au paysage agreste devant le château. En outre, au moment de regagner le jour et ayant cassé les dernières vitres, Ariane voit « les yeux fermés, les longues pierreries qui fouettent [s]es paupières » (M 155), ce qui crée un lien emblématique avec les scènes de la porte, toute inondée qu'Ariane était alors de la lumière (des pierres précieuses) ${ }^{24}$. Que la pierre qui casse les vitres soit appelée "clé » fait de cette délivrance, au plan métaphorique, une seconde transgression de porte, mais dans une logique inverse : la première entraîne la captivité, la seconde inaugure le moment de la liberté reconquise. Les rubis et la couleur rouge jouent également un rôle important, puisqu'ils transposent le meurtre et la cruauté - éléments incontournables de Barbe-Bleue dans sa forme classique - au niveau chromatique. Si la cinquième porte est placée sous le signe du sang ("Le sang coule sous la porte permise », M 138), la dernière porte figure, à travers les diamants, non les affres de la violence, mais la clarté du savoir et, partant, le secret dévoilé de Barbe-Bleue. Tout concourt à penser que le texte souligne la ruse d'un Barbe-Bleue qui a recours à une cruauté plus subtile et de nature psychique dans la mesure où il a soin de marquer déjà une porte permise d'une menace infaillible, d'un terrible mane tekel.

Les couleurs des pierres précieuses, loin de n'être qu'un élément décoratif dans la tradition des contes et de l'esthétique symboliste, sont en relation analogique avec l'acte de la délivrance. Les couleurs < captives > à l'intérieur des chambres et la transformation de celles-ci en éléments de la nature, à l'extérieur, forment une autre délivrance, dans ce cas au niveau chromatique. Il en résulte une cohérence étroite entre les deux premiers actes. Elle est encore renforcée, comme nous l'avons vu, par le rapprochement des deux moments de transgression, étant donné que la clef en or qui ouvre la porte interdite se double de la pierre cassant les vitres et désignée métaphoriquement comme «clef» par Ariane : «Voici la clef de votre aurore » (M 155).

Il nous reste à faire une dernière remarque sur la symbolique des chiffres censée compléter celle des couleurs. Au nombre des portes s'ajoutent les six fenêtres monumentales introduites dans la première indication scénique. Ouvertes au troisième acte, elles signalent la délivrance des six femmes et rappellent bien sûr les portes ouvertes du premier acte. Maeterlinck, appliquant un léger changement au chiffre sept de la tradition tieckienne, fixe donc le nombre des femmes à $\operatorname{six}^{25}$.

${ }^{24}$ Au début de l'acte III, leur présence est rappelée dans l'incipit qui précise le décor («Les pierres éparses scintillaient encore dans les niches de marbre », M 160) et ce sont elles aussi qu'Ariane répand sur ses compagnes.

${ }^{25}$ Il s'agit bien de six femmes chez Maeterlinck (Ariane, Sélysette, Mélisande, Ygraine, Bellangère, et Alladine) et non de sept comme l'affirme Patrick Mahony (Maurice Maeterlinck. Mystic and Dramatist. Washington 1979, 91). 
Le texte a soin de le préciser dès le début quand la voix de la foule dit d'Ariane : « ça fera la sixième »( $M$ 132). Il est intéressant de noter que le texte joue consciemment avec le nombre symbolique de sept. Sélisette (!), en comptant les gardes, se trompe de manière significative : "Un, deux, trois, quatre, six, sept. Mais ils ne sont que sept ! ...» (M 165). L'échec final de la délivrance rend-il impossible l'idéal et le divin que représente le chiffre sept ? Ou scelle-t-il à jamais le destin des femmes, qui exclut (au septième jour et au septième âge) toute rédemption ainsi que tout espoir de trêve de la cruauté de Barbe-Bleue ?

\subsection{Vers une première exégèse du bleu}

Rares sont les études sur Barbe-Bleue qui ne tentent pas d'interpréter la couleur bleue. Le problème, comme le dit fort justement Winfried Menninghaus ${ }^{26}$, réside dans sa plurivalence, dans sa polysémie même : si le bleu est l'azur, il est aussi l'enfer ; s'il représente la fidélité, il peut tout autant tromper. Cette ambiguïté est pertinente par exemple chez Goethe dans sa Farbenlehre. La couleur bleue y est associée à la pureté suprême et à l'énergie, mais elle véhicule en même temps, selon le classique weimarien, quelque chose de sombre, un sentiment de froideur ${ }^{27}$.

Il convient donc, pour Ariane et Barbe-Bleue, d'élargir le champ d'analyse à d'autres œuvres de Maeterlinck. On commence par constater que le bleu, dans Serres chaudes, recueil de poésie publié en 1889 chez Vannier, est une couleur fréquemment mise en scène et liée le plus souvent à des états de mélancolie rêveuse. Dans Serre d'ennui, le bleu est le signe chromatique de l'ennui et du spleen dans la tradition verlainienne évoquée par les célèbres rimes \& cœurpleur > et 〈 cœur-langueur > : «O cet ennui bleu dans le coeur / Avec la vision meilleure / Dans le clair de lune qui pleure / De mes rêves bleus de langueur $»^{28}$. Ce 〈 rêve bleu > n'est pas l'azur, espace poétique par excellence et terme d'un voyage initiatique dont rêve le je mallarméen dans le poème $L$ 'Azur, mais exprime ici l'état de la stérilité, du taedium vitae. À cet égard, on trouve aussi l'expression « esprit bleu » (Âme chaude) ou " cristal bleu de mes lasses mélancolies » (Feuillage du cœur). Ceci étant, le bleu, au même titre que l'infraction au chiffre sept, exprimerait le pessimisme et la torpeur finale de la pièce.

Une autre référence - qui l'est d'autant plus pour Maeterlinck - est la 〈blaue Blume >, emblème de toute une époque romantique de désirs vagues. Déjà associée dans les contes populaires à des trésors cachés, la 〈 hohe lichtblaue Blume >, dans le roman fragmentaire Heinrich von Ofterdingen de Novalis, est un symbole de la connaissance de la nature, de son unité - s'inscrivant dans le pansymbolisme du romantique allemand - et du nosce te ipsum. Le rapprochement s'impose d'autant

${ }^{26}$ Menninghaus (voir note 5) 73.

${ }^{27}$ Goethe, Johann Wolfgang : Zur Farbenlehre. Didaktischer Teil. München 1963, 171.

${ }^{28}$ Maeterlinck, Maurice : Serre d'ennui (première strophe). Dans : Poésies complètes. Bruxelles 1965, 97. 
plus que, rappelons-le, Maeterlinck est un grand lecteur de Novalis, dont il a traduit notamment les Fragmente ${ }^{29}$. Aussi peut-on relever sa dette envers le symbolisme chromatique d'un Ruysbroeck évoquant la descente de l'âme de Dieu en bleu$\operatorname{ciel}^{30}$. Cette connotation persiste également chez Baudelaire quand il évoque le «bleu mystique », précisément dans le contexte de la mort et de l'ange qui ouvre les portes, dans La mort des amants des Fleurs du mal ${ }^{31}$. Le bleu devient alors la couleur de la recherche métaphysique, du savoir sur l'essence du monde, de l'immanent certes, mais surtout du transcendant, un point idéal auquel aspirent tous les désirs. Cette lecture peut être étayée par L'Oiseau bleu (1909), autre conte en forme de pièce de théâtre, en ce sens que l'oiseau bleu, pour revêtir les mêmes qualités que la fleur bleue, y est défini comme «le grand secret des choses et du bonheur $»^{32}$.

Concluons donc que c'est surtout la fonction de la recherche de l'absolu, du mystère de l'essence divine ou d'une force transcendantale qui serait à retenir pour Maeterlinck. Ce qui est la quête de la fleur bleue ou de l'oiseau bleu devient la quête du secret de Barbe-Bleue, sujet auquel nous reviendrons au dernier chapitre. Il est révélateur que l'alternative finale pour les premières épouses, c'est-à-dire de s'en aller avec Ariane ou de rester avec Barbe-Bleue, s'exprime une fois de plus au niveau chromatico-symbolique dans la mesure où il s'agit d'un choix entre deux sortes de bleus, entre « la campagne bleue », l'azur "d'un monde inondé d'espérance » que va offrir Ariane (M 174s.) et le bleu de l'engourdissement léthargique qu'est le château de Barbe-Bleue. Nous connaissons la fin : fatalement, le bleu du mari l'emportera.

\section{Le destin des femmes et la délivrance}

\subsection{De la femme forte comme filiation perraultienne à l'emprise secrète de Barbe-Bleue}

La majeure partie de la critique s'entend sur le point de considérer la pièce maeterlinckienne comme l'un des premiers textes qui mette en scène une femme

${ }^{29}$ Pour une étude détaillée sur l'influence allemande (et de Novalis) dans l'œuvre de Maeterlinck cf. Gorceix (voir note 9).

${ }^{30} \mathrm{cf}$. Hanak, Miroslav John : Maeterlinck's symbolic drama. A leap into transcendence. Louvain 1974, 52s. Voir pour la descente de l'âme en bleu et les pierres précieuses Versluys, L. (éd.) : L'Ornement des noces spirituelles de Ruysbroeck l'Admirable, traduit du flamand et accompagné d'une introduction par Maurice Maeterlinck. Bruxelles 1990, 45 .

${ }^{31}$ Baudelaire, Charles : Les Fleurs du mal. éd. John E. Jackson. Paris 1999, 182.

${ }^{32}$ Maeterlinck, Maurice : Théâtre 2. Bruxelles 1999, 312. Voir à ce sujet Gorceix, Paul : L'Oiseau bleu. Un Märchen initiatique. Dans : Le Symbolisme en Belgique ou l'éveil à une identité culturelle. Paris 2008, 243-266. L'auteur rapproche le symbole de l'oiseau bleu du mysticisme (des romantiques allemands), de la recherche novalisienne du moi transcendental, du grand sujet qu'est l'inconnaissable chez Maeterlinck. 
forte sachant se libérer de Barbe-Bleue. Toujours est-il qu'il faudrait préciser que ce revirement s'annonce déjà chez Perrault. La deuxième moralité («Autre moralité »), en effet, nous apprend sur un ton humoristique que Barbe-Bleue est une figure masculine révolue. D'ores et déjà, l'homme va « filer doux » ${ }^{33}$ devant sa femme, et il est très difficile de savoir qui est le véritable maître à la maison. La domination du mâle < bleu > est subtilement minée si bien que, contre toute apparence, la femme règne en secret sur l'espèce des barbus (et de n'importe quelle couleur ...). Quant au rapport conclusif avec le conte, l' « Autre moralité » n'est pas sans poser problème. À moins qu'on ne veuille considérer la domination secrète de la femme dans le fait de transgresser l'interdiction du mari, doit-on y voir une parodie de la moralité en tant que telle (moralité autre) ou plutôt un < Verfremdungseffekt, par rapport à la cruauté extrême du conte ? À cet égard, il est significatif de noter que, comme le précise Mererid Puw Davies ${ }^{34}$, cette moralité n'était pas incluse dans le manuscrit de 1795, mais qu'elle n'apparut que dans la première édition en 1797.

La question que la deuxième moralité soulève, c'est-à-dire l'existence possible d'un maître secret féminin, paraît distinguer chez Maeterlinck deux types de femme : le modèle 〈 progressiste > d'Ariane, forte et supérieure à Barbe-Bleue, et celui des premières femmes, faibles et sans volonté intrinsèque, même auprès d'un mari garrotté et blessé. Ariane et Barbe-Bleue étant souvent qualifié de pièce féministe, la torpeur finale des cinq autres femmes de Barbe-Bleue, réduites à l'existence de marionnettes fantomatiques, doit malgré tout être prise en considération. Quoique l'affrontement entre homme et femme - thème majeur du mythe - se joue sans morts chez Maeterlinck, Barbe-Bleue, faible tout au long de la pièce et fait prisonnier à la fin par le peuple furieux, triomphe en parvenant à retenir les cinq autres femmes qui, au lieu de suivre Ariane vers la liberté, restent auprès de lui. Mais n'oublions pas que Barbe-Bleue n'est plus Barbe-Bleue, tout dépourvu qu'il est in fine du principe de sa force et de sa virilité qu'est la barbe. Car la peur disparaît au moment où la barbe est rasée - dernière ruse de Barbe-Bleue n'ayant gardé que son manteau bleu - et que cette castration faciale semble le priver (apparemment ...) de tout son pouvoir de mâle. Maeterlinck hérite alors de la tradition de la barbe bleue comme stigmatisation et « signum mali » ${ }^{35}$ et annonce de même les figures des Barbe-Bleues sans barbe du XX $X^{e}$ siècle. Il s'agit en fait d'une solution lapidaire du dilemme de Barbe-Bleue : si, déjà chez Perrault, la barbe terrifie les possibles épouses, pourquoi ne pas avoir recours au barbier ... ? ${ }^{36}$ Malgré le clin d'œil à cette solution dans Ariane et Barbe-Bleue, il n'empêche que l'absence de la barbe, ajoutée à celle de la parole (Barbe-Bleue réapparaît sur scène à la fin de l'acte III, mais est complètement muet), n'est qu'un leurre. Le pouvoir sur les premières épouses reste intact, lesquelles semblent être vouées à un

${ }^{33}$ Perrault, Charles : La Barbe Bleue. Dans : Contes. Paris 1991, 129.

${ }^{34}$ Davies (voir note 22) 46.

${ }^{35}$ Szczepaniak (voir note 11) 102.

${ }^{36}$ Menninghaus (voir note 5) 78. 
enfermement éternel. Étant aussi insatiable en matière de femme et aussi stérile, Barbe-Bleue se transforme une fois de plus en double de Don Juan. Ariane n'est pas sans le remarquer et dit qu'il veut, non le célèbre « mil e tre >, mais au moins " cent femmes » (M 163). Celle-ci, une créature d'exception dans le monde féminin de Maeterlinck, est la seule des épouses qui échappe au patriarcat du pèreépoux. C'est déjà elle, rappelons-le, qui s'est arrogée le pouvoir de pardonner (à l'inverse du pardon refusé de Barbe-Bleue) alors que les autres femmes, toujours passives, n'ont pas cherché à s'évader de la prison souterraine puisque «c'est défendu » (M 150). La première désobéissance à la porte n'est pas suivie d'une autre. Voilà comment le texte anticipe sur la victoire finale de Barbe-Bleue sachant défendre ses premières épouses contre l'intruse.

La couleur bleue, liée dans le cas d'Ariane à la recherche du secret, symbolise pour les autres femmes leur fidélité aveugle à Barbe-Bleue : celui-ci devient pour elles cette figure du Destin que lui reconnaît, par exemple, Anne Pourtois ${ }^{37}$. Sa force psychologique se trouve figurée, par le principe du paysage intérieur si cher à l'auteur de Pelléas et Mélisande ${ }^{38}$, dans le château enchanté. En effet, le symbolisme du lieu se configure en relation secrète avec la psychologie des personnages : la contrainte matérielle de rester (à cause du pont levé) exprime le pouvoir quasiment magnétique du mari, dont, malgré ses atrocités, une image transfigurée perdure dans l'imagination des premières femmes s'opposant toutes à ce que le peuple le tue. Il est donc judicieux de placer, selon Jürgen Wertheimer ${ }^{39}$, la pièce maeterlinckienne - avec Offenbach (Barbe-Bleue, 1866) et Bartók (Le Château de Barbe-Bleue, 1911) - sous l'enseigne de "Blaubarts Triumph » et d'y reconnaître une orientation décisive que prend le mythe au $X^{\mathrm{e}}$ siècle. On peut résumer un aspect central de cette nouvelle orientation avec Davies : "But the transformation of Bluebard into a Symbolic Order means that violence has become psychological and thereby normalized as well as made more invisible and pervasive $»^{40}$. En transformant la cruauté physique de Barbe-Bleue en pression psychologique subtile, Maeterlinck crée une pièce qui a bien sa place au seuil de la modernité. Dès lors, le meurtre est beaucoup plus celui d'une attaque sournoise à la personnalité féminine que d'atroces brutalités infligées aux corps. Ariane est la seule à casser la vitre de la prison souterraine alors que les premières femmes ne désobéissent pas une seconde fois. Toute «l'efficacité > du nouveau Barbe-Bleue consiste à ne plus devoir tuer les femmes, mais d'avoir réussi à tuer à jamais leur curiosité.

${ }^{37}$ Pourtois (voir note 23) 73.

${ }^{38}$ Pour ce procédé typique de l'art théâtral de Maeterlinck, on peut se référer utilement à McGuiness, Patrick : Maurice Maeterlinck and The Making of Modern Theatre. Oxford 2000.

${ }^{39}$ Wertheimer (voir note 20) 114.

${ }^{40}$ Davies (voir note 22) 233. 


\subsection{La quête du savoir et la délivrance sous le signe du double}

Nous avons fait allusion, au cours des deux derniers chapitres, à la mission salvatrice d'Ariane. Il est indispensable de revenir sur la motivation primaire de cette délivrance, c'est-à-dire la quête du savoir autour du secret de Barbe-Bleue. La curiosité féminine que Perrault stigmatise avec un clin d'œil, devient chez Maeterlinck une sincère quête du savoir; ou, en d'autres termes, tandis que le narrateur perraultien critique - avec une forte dose d'humour - la curiosité excessive des femmes dans la première morale, Maeterlinck valorise la quête du savoir et la considère comme vitale dans un univers régi par la peur et la passivité. "Tout ce qui est permis ne nous apprendra rien » (M 134), voilà l'énoncé gnomique érigé en principe irrévocable. Ariane, à la recherche du secret de son mari, jette donc les six clefs d'argent pour ne garder que celle en or qui lui est défendue, et laisse, par conséquent, sa nourrice ouvrir toutes les autres portes puisqu'elles ne renferment aucun savoir. Or, parmi toutes les pierres précieuses derrière les portes, les diamants de la sixième sont les seuls qui intéressent Ariane, et la pluie de pierres précieuses s'étant ouverte, ils mènent significativement à la porte fatale. L'éclat des diamants représentant le savoir, la clarté de la révélation et la septième porte close cachant le secret du mari sont donc liés de manière figurative.

Tout au début, dans sa première intervention, Ariane énonce la règle capitale : «Il faut d'abord désobéir ; c'est le premier devoir quand l'ordre est menaçant et ne s'explique pas » (M 133). Le devoir n'est pas l'obéissance, mais l'exact contraire. La désobéissance se dresse non seulement contre l'ordre impérieux (du mari, de la société), mais en même temps contre le caractère inexplicable et mystérieux de la défense. Il n'est pas anodin que cette quête du savoir soit réaffirmée au moment où la transgression est découverte. Les alexandrins très réguliers confèrent à ce moment clé plein d'intensité et de pathos une dimension solennelle, consacrée :

« [Ariane]: Le bonheur que je veux ne peut vivre dans l'ombre.

[Barbe-Bleue] : Renoncez à savoir et je puis pardonner ...

[Ariane] : Je pourrai pardonner lorsque je saurai tout » (M 142s.).

La lutte entre le pôle masculin et féminin, entre l'ombre du secret et la clarté du savoir, évolue ici en un conflit de rôle, en une revendication de part et d'autre pour l'instance octroyant le pardon. Dans la version maeterlinckienne, le bonheur nuptial réside donc bien dans la découverte du secret de Barbe-Bleue : « Il m'aime, je suis belle et j'aurai son secret »( $\mathrm{M}$ 133). La libido sciendi frôle l'interprétation freudienne du mythe, qui comprend le franchissement de la porte interdite en tant qu'initiation sexuelle de la nouvelle mariée.

Conformément aux schémas proppiens, le conte finit chez Perrault avec la sanction, la punition du méchant (poignardé par les deux frères), la récompense des héros (l'épouse de Barbe-Bleue achète des charges de capitaine pour eux) et un 
mariage (la femme se remarie avec un "fort honnête homme ${ }^{41}$ ). Le conte aura son happy end ${ }^{42}$. L'action chez Maeterlinck, néanmoins, semble rebondir au moment où la porte défendue est ouverte et que Barbe-Bleue apparaît pour la punition. La première 〈 délivrance > d'Ariane (ici par le peuple) se situe à la fin du premier acte, mais, comme l'indique le sous-titre, elle est inutile à ses yeux (« Il [Barbe-Bleue] ne m'a fait aucun mal ", M 144). Le deuxième acte commence là où finit Perrault : avec la délivrance in extremis d'Ariane. Bouclant la boucle, le troisième acte thématisera précisément la délivrance des autres femmes, tout aussi inutile puisqu'elles restent auprès du mari. Il apparaît que la délivrance sous le signe du double est précédée d'une double transgression : celle, au début devant la porte défendue, celle, un acte plus tard, devant la vitre de la grotte souterraine. Il s'y ajoute qu'après les délivrances proprement dites suit, au début de l'acte III, une autre délivrance sur le plan figuratif et esthétique. Car Ariane, soucieuse de récréer la beauté des premières femmes, s'emploie à «délivrer la lumière " (M 161) en dénouant les tresses de Mélisande, et délivre de même " deux rayons de bonheur » (M 162) des manches de Sélysette.

Rappelons dans ce contexte que la figure d'Ariane constitue, dans la pièce de Maeterlinck, une double inversion par rapport à la tradition : d'une part, au lieu d'être délaissée comme son pendant mythologique, elle se sépare résolument de son mari ; d'autre part, en comparaison avec l'épouse perraultienne, elle évolue d'un sujet passif qui doit être délivré en figure active de sauveur, en délivrant non seulement les autres femmes passives, mais également Barbe-Bleue, que le peuple lui livre captif, c'est-à-dire dans le même état que l'étaient ses épouses auparavant. Ainsi l'acte de la délivrance est-il, par rapport à l'objet, double aussi : des femmes, Ariane passe à Barbe-Bleue, lequel, quant à lui, devient le captif des mêmes femmes qu'il a naguère enfermées. Contrairement à la première délivrance d'Ariane qu'entreprend la collectivité du peuple, la deuxième renferme une dimension par essence tragique d'autant que les premières femmes sont incapables de se séparer de l'homme qui les retient captives. De la délivrance inutile dans le sens de 〈non nécessaire `, on en vient à l'acception 〈 en vain 〉. Il est révélateur que, comme dernier geste, Ariane ferme la porte en haussant les épaules et refait exactement le même mouvement que tout à la fin de l'acte I, où elle a fermé la porte défendue : début et fin de la délivrance se retrouvent.

La porte de la transgression et de la liberté une fois ouverte, elle se referme à la fin pour que tout - et pour toujours - puisse recommencer dans ce huis-clos sartrien qu'est l'enfer de Barbe-Bleue : "Eh bien, continuons ... ». Ce que Monika Szczepaniak appelle «Erlösung aus dem Blaubart-Gefängnis » ou « auf der Farbe blau aufbauender Erlösungsdiskurs ${ }^{43}$ échoue complètement chez Maeterlinck et

${ }^{41}$ Perrault (voir note 33) 128.

${ }^{42}$ Le nouvel époux « lui [à la femme] fit oublier le mauvais temps qu'elle avait passé avec la Barbe bleue » (ibid.).

${ }^{43}$ Szczepaniak (voir note 11) 283. 
confirme le caractère proprement dramatique (et pessimiste) de sa vision de Barbe-Bleue.

En guise de conclusion, revenons brièvement à la figure du double qui semble être le pivot autour duquel tourne Ariane et Barbe-Bleue. Tout est donc double chez Maeterlinck : double délivrance inutile, double transgression, double clef, double conception de la femme (faible et forte) et de Barbe-Bleue (avec barbe ou rasé, soit magnétique ou apparemment impotent) ; double regard, enfin, sur le conflit et le pouvoir des pôles masculin et féminin, d'une Ariane dominante et d'un Barbe-Bleue qui, en retenant ses premières épouses, triomphe quand même.

Le motif du double relève d'une part de la structuration narrative sous l'enseigne d'une esthétique d'écho telle que les renvois de la chromatique symbolique ou la stichomythie typiquement maeterlinckienne. D'autre part, elle conduit à une complexification savamment construite du mythe de Barbe-Bleue sur plusieurs plans : le croisement avec d'autres mythèmes, le jeu de miroir avec le modèle mythologique d'Ariane ou celui avec tel ou tel élément de la tradition perraultienne, pour ne pas oublier la quête du secret et la recherche métaphysique. À travers cette condensation centrifuge, l'apport symboliste de Maeterlinck, placé au seuil de la modernité, est fondamental pour le destin fécond réservé à Barbe-Bleue au cours $\mathrm{du} \mathrm{XX}^{\mathrm{e}}$ siècle.

\section{Resumé}

Maurice Maeterlinck oriente la tradition de Barbe-Bleue dans plusieurs directions inédites : il la rattache à d'autres mythèmes, surtout en la combinant avec la figure d'Ariane ; et il adapte des éléments traditionnels (les premières femmes ne sont pas mortes mais prisonnières) ou en développe d'autres à la lueur de l'esthétique symboliste (couleurs, portes et pierreries). La curiosité féminine que réprouve Perrault avec un clin d'œil, devient une véritable quête du savoir. Ariane et Barbe-Bleue est régi par une structure binaire : une double délivrance est précédée de deux transgressions. Comme le symbolisent les deux clés, la pièce montre le caractère multifacétique du masculin et du féminin : une Ariane femme forte, progressiste, contraste avec les autres femmes anxieuses et faibles. Barbe-Bleue, sans barbe et captif à la fin, l'emporte dans la mesure où il arrive à retenir les premières femmes. La pièce de Maeterlinck peut être considérée comme une œuvre clé qui ouvre la voie aux adaptations modernes du $\mathrm{XX}^{\mathrm{e}}$ siècle.

\section{Abstract}

Maurice Maeterlinck develops the Bluebeard tradition in many regards: by linking it with other myths, especially - in a constant play of mirrors - with the mythological model of Ariane, by rethinking some of the traditional elements (the first female victims are not dead but prisoners) or by reworking them in light of symbolist aesthetics (colours, doors with hidden jewelry). The curiosity which Perrault stigmatizes (tongue in cheek) turns into a serious metaphysical inquiry, a real quete du savoir. The underlying structure of Ariane et Barbe-Bleue can be defined as a binary one: a double deliverance responds to a double transgression. As symbolized by the two keys, the plot unveils the double faces both of the 
female and the male principles. The play contrasts the strong and 'progressive' woman, Ariane, with the weak and anxious other wives. Bluebeard, beardless and captured in the end, prevails as far as he can deter his former wives from following Ariane into liberty. Maeterlinck's version can be considered as a turning point, giving rise to the modern avatars of Bluebeard.

\section{Zusammenfassung}

Maurice Maeterlinck entwickelt die Blaubart-Tradition in diversen Aspekten entscheidend weiter: durch den Einbezug mehrerer anderer mythologischer Themen, vor allem mit der Figur der Ariane, aber auch durch die Adaption traditioneller Elemente (die ersten BlaubartFrauen sind lediglich Gefangene) oder deren poetisch-symbolistischer Ausgestaltung (Farben, Türen mit versteckten Edelsteinen). Die weibliche Neugier, von Perrault noch mit einem Augenzwinkern mißbilligt, wird zu einer metaphysischen Suche des Ursprungs. Die Struktur von Ariane et Barbe-Bleue ist die einer spiegelbildlichen Doppelung: der doppelten Befreiung geht eine zweimalige Mißachtung des Verbots voraus. Die zwei Schlüssel verweisen auf die Janusköpfigkeit des Männlichen und Weiblichen: eine starke und fortschrittliche Ariane kontrastiert mit den schwachen, schemenhaften Frauen. Blaubart, am Ende bartlos und gefangen, behält insoweit die Oberhand, als er seine ersten Frauen zurückzuhalten vermag. Ariane et Barbe-Bleue kann als Schlüsselstück gesehen werden, das viele neuere Bearbeitungen vorwegnimmt. 